\title{
The Value of Doppler Venous Pressure Index in Chronic Venous Disease of the Lower Limbs
}

\author{
Tommaso Spina ${ }^{\mathrm{a}}$, Elio Ferlaino ${ }^{\mathrm{a}}$, Daniele Pontello ${ }^{\mathrm{a}, \mathrm{b}}$, Leonardo Corcos ${ }^{\mathrm{a}}$
}

\begin{abstract}
Background: The diagnostic evaluation of chronic venous disease (CVD) of the lower limbs (LLs) is mainly based on duplex ultrasound (DUS). Invasive venous pressure measurements (VPMs) are seldom performed in specialized centers with still controversial results. The noninvasive Doppler method has not been accepted as a validate method as the emerging values are influenced by several environmental and biological factors, and do not correspond to hydrostatic pressure. Therefore, these values will be defined as venous pressure index (VPI). In our previous experience, the VPI was accepted by patients in the daily practice, and the VPI was significantly correlated with $\mathrm{C}$ of C.E.A.P. $(\mathrm{P}<0.05-0.0001)$ and furnished useful hemodynamic information. This study was to verify the role and clinical implications of the VPI measurements in the pathophysiology and diagnostics of superficial CVD of the LL.
\end{abstract}

Methods: The 2,098 LLs of 1,049 patients affected with CVD and/ or other pathologies of the LL were subjected to DUS investigations. The 1,212 LLs of 606 patients were subjected to VPI measurements which were compared with 162 normals. LLs with venous malformations and deep venous disease were excluded owing to the small number of cases. Standing and ambulatory VPIs were detected by Doppler method at the ankle in correspondence of the greater saphenous vein (GSV), smaller saphenous vein (SSV) and posterior tibial vein (PTV). The VPI mean values were correlated with the site, extension and various combinations of reflux $(\mathrm{R})$ and analyzed.

Results: Standing VPI is significantly related with the site, extension and combinations of venous $\mathrm{R}$, while the ambulatory VPI did not furnish significant data. Mean VPI values: GSV > SSV; GSV with isolated $\mathrm{R}$ at the leg $>\mathrm{GSV}$ at the thigh; additional $\mathrm{R}$ in perforators increases VPI in all the superficial districts; superficial $\mathrm{R}$ increases VPI in PTV.

Manuscript accepted for publication September 18, 2015

avascular Laboratory of the Prosperius Institute of Firenze, V. le Fratelli Rosselli n. 62, 50123 Firenze, Italy

${ }^{b}$ Corresponding Author: Daniele Pontello, Via D. Chiesa n. 22, 33038 San Daniele Del Friuli (Ud), Italy. Email: ciuto77@virgilio.it

doi: http://dx.doi.org/10.14740/jcs282e
Conclusions: Standing VPI is the significant expression of the hemodynamic alteration in the various venous districts. R of GSV at the leg and in perforators is related with higher VPI and severity of the disease. Superficial venous hypertension is correlated with deep venous hypertension. Doppler VPI measurement is a simple repeatable, highly predictive investigation; it furnishes precious hemodynamic information for selection, treatment and follow-up of patients.

Keywords: Venous; Pressure; Hypertension; Varicose; Veins; Insufficiency; Elastic compression; Stockings

\section{Introduction}

Many authors in the past, from 1733 up to now, performed venous pressure measurements (VPMs) by means of invasive methods which have been partially resumed by Hojensgard and Sturup [1].

The first proposal for doing the examination by noninvasive Doppler method was presented in 1975 by Gaylis [2] and further on by Bartolo in 1977 [3]. In 1985 Bartolo et al published a study of 8,742 lower limbs (LLs) affected with various venous disease which were compared with normal limbs. The emerged data were of a high statistical significance [4].

The modern hemodynamic evaluation of chronic venous disease (CVD) of the LL is mainly based on duplex ultrasound (DUS) examination. Hemodynamic investigations as VPM and plethysmography are systematically performed in few centers [5-8]. The majority of the authors accept the invasive VPM which furnish hydrostatic values but do not trust in the results obtained by Doppler method, as they are influenced by many different anatomical, functional, biological, mechanical and/or environmental factors. These factors of variation cannot be completely investigated and analyzed. For this reason, the values resulting from Doppler method should be better defined as venous pressure index (VPI).

We have been using the noninvasive VPI measurement in the differential diagnosis of the majority of the clinical cases affected with various venous diseases of the LL since 1985. Since 1996, we started the use of a phlebologic software for the informatic filing of all the cases studied in our Vascular Laboratory [9]. The emerging data and the experience acquired clearly demonstrated that the VPI measurements could give 
Table 1. Distribution of the Patients Subjected to VPI Doppler Measurements

\begin{tabular}{lll}
\hline & N & \% \\
\hline Patients examined & 1,049 & 100 \\
Patients without CVI & 162 & 15.4 \\
Patients with CVI & 887 & 84.5 \\
Average age & $52.4 \pm 16.2$ & \\
Male & 273 & 26.0 \\
Female & 776 & 74.0 \\
Right & 554 & 52.8 \\
Left & 495 & 47.1 \\
\hline
\end{tabular}

CVI: chronic venous insufficiency.

useful information concerning the severity and pathophysiology of CVD, and several therapeutic implications were noted. We often decided where and how to extend superficial and/ or deep veins surgery and/or endovascular treatments, as endovenous laser and sclerotherapy of superficial venous insufficiency of the LL, for the patient's follow-up and for a more precise selection of the class of compression stockings [10], which rendered the patients more compliant than in the past [11], on the bases of the VPI results.

The aim of this study was to verify the role and significance of the VPI measurements in the pathophysiology and diagnostics of superficial CVD of the LL and their clinical and therapeutic implications.

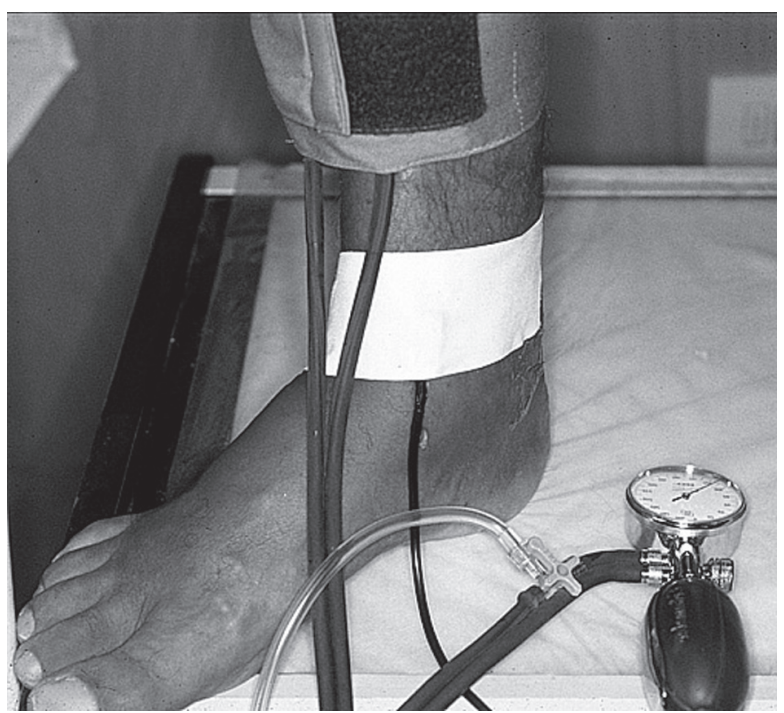

Figure 1. VPI Doppler measurement on a right LL of a patient affected with CVI. Placement of the pneumatic cuff and of the flat probe of 8 $\mathrm{MHz}$ by plaster is visible.

\section{Materials and Methods}

In the period comprised between 1996 and 2002, 2,098 LLs of 1,049 patients affected with CVD and/or other pathologies of the LL were studied by clinical and DUS investigations. The distribution of the cases is reported in Table 1.

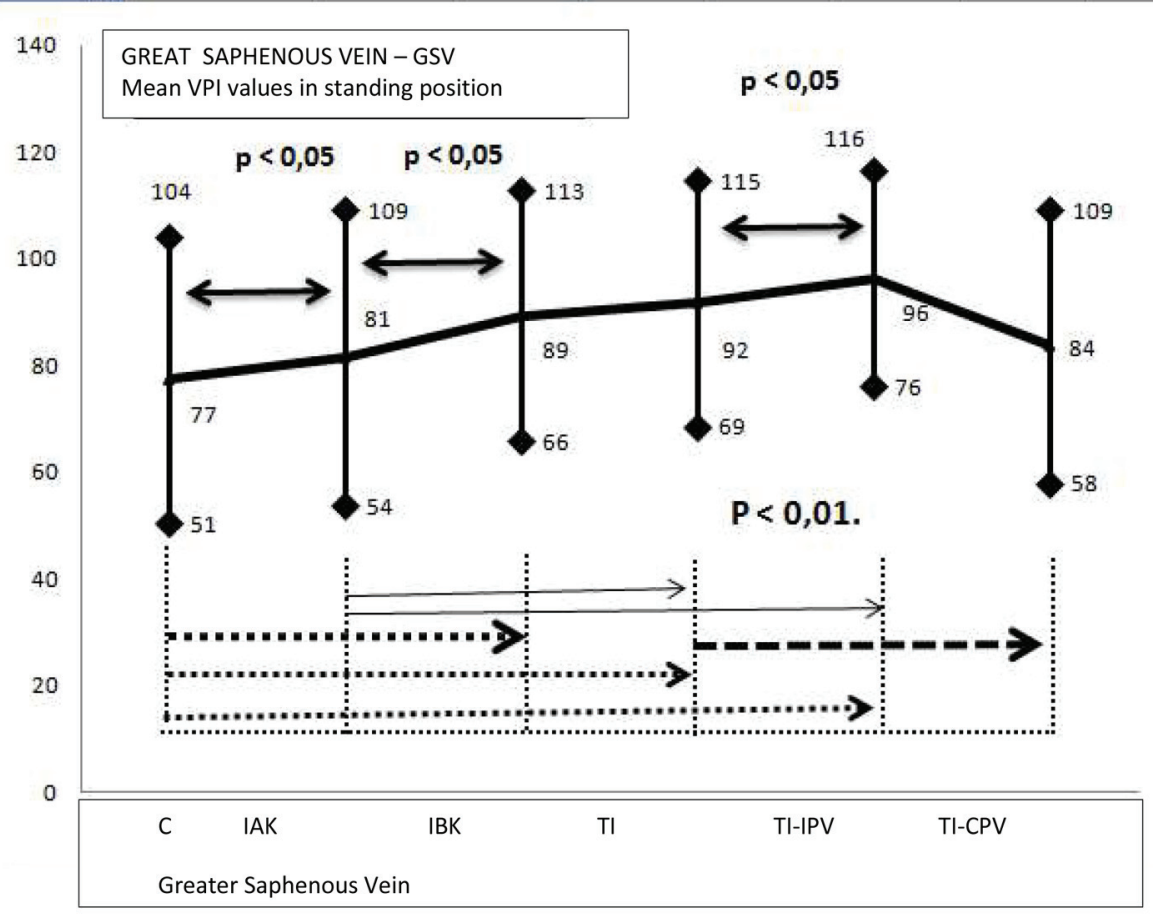

Figure 2. VPI values of the GSV measured in standing position. C: competent; IAK: incompetent above knee; IBK: incompetent below knee; TI: totally incompetent; TI-IPV: totally incompetent and incompetent perforating veins; TI-CPV: totally incompetent and competent perforating veins. 
Table 2. Statistical Analysis of the Standing PVI Values of the GSV Measured in Standing Position

\begin{tabular}{llllll} 
& \multicolumn{5}{c}{ Student's $t$-test of the GSV of standing PVI values } \\
\cline { 2 - 5 } & GSV C & GSV IAK & GSV IBK & GSV TI & GSV TI-IPV \\
\hline GSV IAK & N.S. & & & & \\
GSV IBK & 0.0002 & 0.0327 & & & \\
GSV TI & 0.00001 & 0.00001 & N.S. & 0.0048 & \\
GSV TI-IPV & 0.0216 & N.S. & N.S. & 0.0202 & 0.00001 \\
GSV TI-CPV & 0.00001 & 0.00001 & 0.0344 & 0.034 \\
\hline
\end{tabular}

C: competent; IAK: incompetent above knee; IBK: incompetent below knee; TI: totally incompetent; TI-IPV: totally incompetent and incompetent perforating veins; TI-CPV: totally incompetent and competent perforating veins.

Both cross and longitudinal USD scanning (Sonoscape 1000; SonoMed Bio, Inc., Bayamon, Puerto Rico ${ }^{\circledR}$ ) were performed at the following venous sites: common femoral vein, popliteal vein, deep veins of the calf, posterior tibial vein (PTV) at the ankle, sapheno-femoral and sapheno-popliteal junctions, greater saphenous vein (GSV) at the junction, thigh and leg, smaller saphenous vein (SSV) at the junction and at the middle calf. The site and the time of reflux (R) were determined [9]. $\mathrm{R}$ with a time of duration of more than $1 \mathrm{~s}$ was considered pathological $[12,13]$. The several diameters were calculated for clinical purposes but the resulting values were not correlated with the VPI measured in the various districts nor analyzed. Perforating veins (PVs) were also investigated and the presence and time of $\mathrm{R}$ were detected in the incompetent ones.

In 1,212 LLs of 887 patients with CVI and 162 normal LLs which were studied in our Vascular Laboratory, the noninvasive VPI measurements were systematically performed. LLs affected with venous malformations, deep venous thrombosis or superficial phlebitis were excluded. The number of the examined LLs affected with deep VI was too small, the statisti- cal analysis appeared to be scarcely significant and they were excluded too.

The VPI values were detected by Doppler method (Dop 2000; Cardioline Milano, Italy ${ }^{\circledR}$ ) in standing position and after 10 tip-toeing exercises (ambulatory VPI) and the differential values were calculated in three venous districts: GSV, SSV and PTV $[3,4]$.

The VPI measurement is performed as follows. The patient is placed over a platform with banister in order to facilitate the standing position and the tip-toeing exercise. A pneumatic cuff of a sphygmomanometer is located at the middle calf and fixed with plaster. A Doppler $8 \mathrm{MHz}$ probe, which can be flat (Fig. 1) or cylindrical, is placed at the ankle level on the skin above the great GSV, the SSV and the PTV. The patient is placed in standing position, and the cuff is inflated up to 120 $\mathrm{mm} \mathrm{Hg}$. The following description of the method belongs to the original papers from Bartolo et al [3, 4]. "Deflating the cuff we can hear at a certain point a blowing noise. The start of their noise indicates the venous pressure and it is the point where the venous flow re-appears being the pressure higher than the ex-

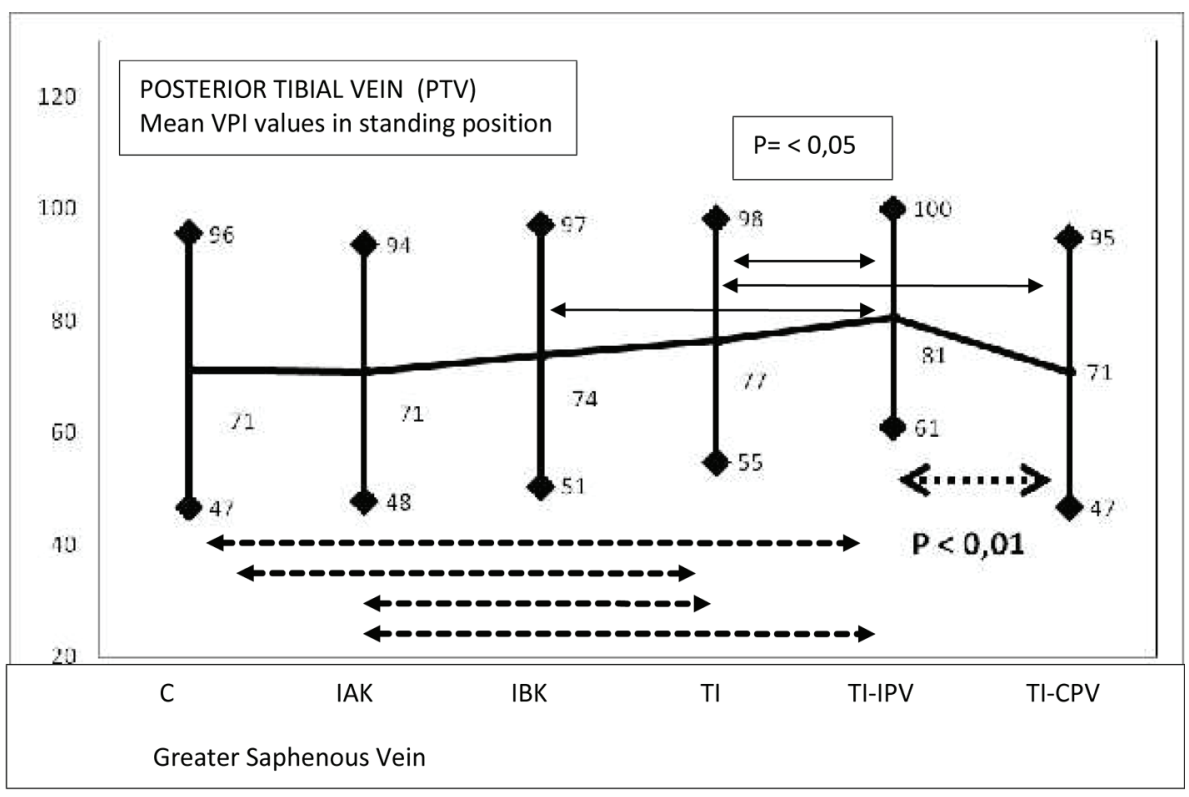

Figure 3. VPI values of the PTV measured in standing position. C: competent; IAK: incompetent above knee; IBK: incompetent below knee; TI: totally incompetent; TI-IPV: totally incompetent and incompetent perforating veins; TI-CPV: totally incompetent and competent perforating veins. 
Table 3. Statistical Analysis of the Standing PVI Values of the PTV in Relation With the Extension of the GSV Valvular Incompetence Measured in Standing Position

\begin{tabular}{llllll} 
& \multicolumn{5}{c}{ Student's $t$-test of the PSV of PVI standing values } \\
\cline { 2 - 5 } & GSV C & GSV IAK & GSV IBK & GSV TI & GSV TI-IPV \\
\hline GSV IAK & 0.844 & & & & \\
GSV IBK & 0.367 & 0.356 & 0.381 & & \\
GSV TI & 0.001 & 0.010 & 0.406 & 0.030 & \\
GSV TI-IPV & 0.897 & 0.976 & 0.034 & 0.035 & 0.00001 \\
GSV TI-CPV & 0.0001 & 0.00001 & & & \\
\hline
\end{tabular}

C: competent; IAK: incompetent above knee; IBK: incompetent below knee; TI: totally incompetent; TI-IPV: totally incompetent and incompetent perforating veins; TI-CPV: totally incompetent and competent perforating veins.

isting one in the cuff, as it occurs when measuring the arterial pressure". The same measurement is performed three times during the diagnostic session in order to verify the reproducibility of the values [4]. Now the patient is asked to perform 10 tip-toe movements by lifting the body on the forward feet, one every second. The inflating-deflating and Doppler detection maneuvers are repeated according with the previous description immediately after exercise.

The PVs function was examined on the basis of the Bjordal principle [14]. He used an invasive method and demonstrated that the elimination of the superficial $\mathrm{R}$ into the saphenous vein could bring back an incompetent PV to a normal function. In our practice, the elimination of the superficial $\mathrm{R}$ into the saphenous vein was performed by applying a rubber tie above the PV subjected to DUS examination in order to verify which PV remained incompetent or was normalized. The presence of $\mathrm{R}$ was detected by peripheral compression maneuver and/or by active muscular contraction of the foot.
The findings made it possible to understand whether the $\mathrm{R}$ detected into the PV was due to a primary venous dilatation and valvular incompetence or to the R detected into GSV. In some cases, the application of the rubber tie at the thigh or at the leg restored a normal flow in the examined PV. In other cases, the PV remained incompetent.

The PVI values are expressed in $\mathrm{mm} \mathrm{Hg}$. All the data obtained were collected by the phlebologic software [9]. The mean values obtained were analyzed by Student's $t$-test with standard deviation and by Bayesian analysis.

\section{Results}

\section{PVI in standing position}

The distribution of the VPI values obtained on the GSV district

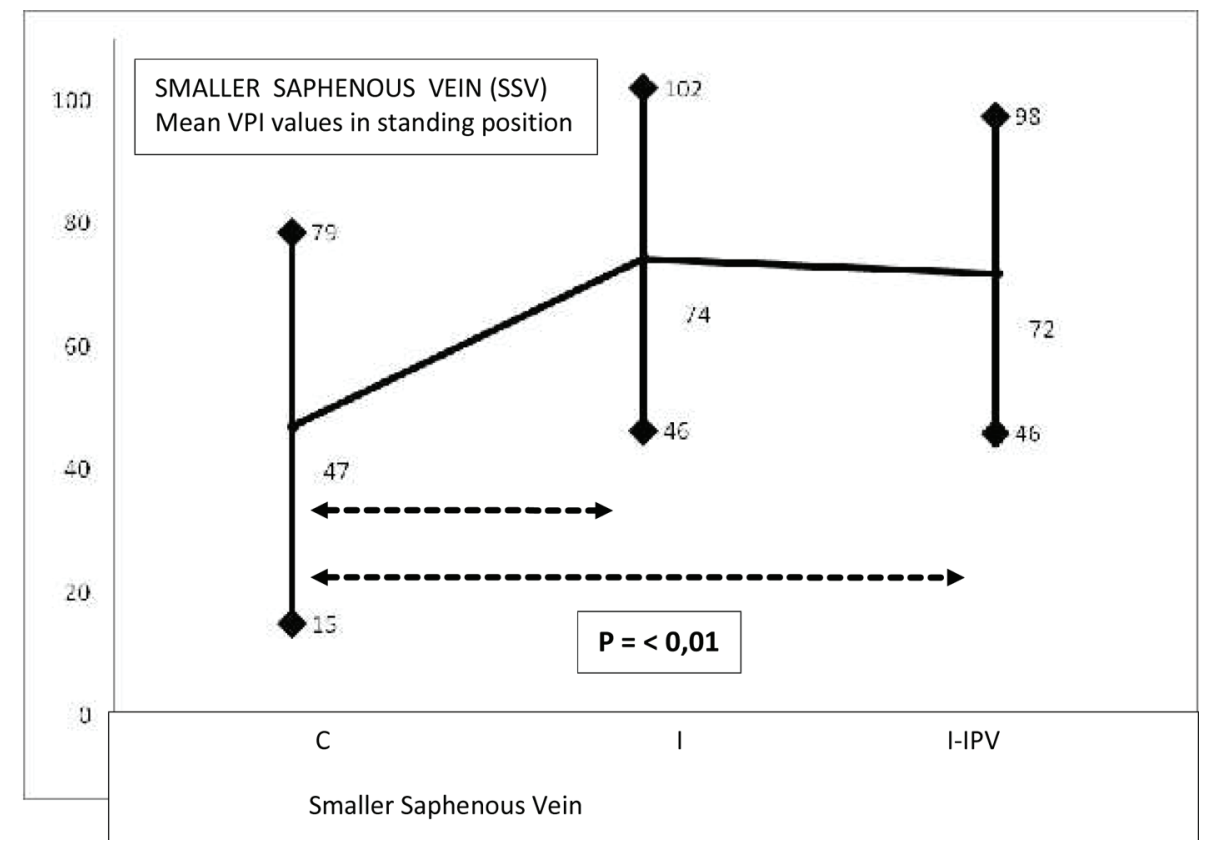

Figure 4. VPI values of the SSV measured in standing position. C: Competent, I: incompetent; I-IPV: incompetent with incompetent perforating veins. 
Table 4. Statistical Analysis of the Standing PVI Values of the SSV Measured in Standing Position by Student's $t$-Test

\begin{tabular}{llr} 
& \multicolumn{2}{c}{ SSV } \\
\cline { 2 - 3 } & C SSV & I SSV IPV \\
\hline SSV C & 2.09 E -24 & 5.27 E - 43 \\
\hline
\end{tabular}

The zeros preceding the reported values are 24 in the first column and 43 in the second $(t<0.01)$. SSV: smaller saphenous vein; C: competent; I: incompetent; IPV: incompetent perforating veins.

is shown in Figure 2. The results of the statistical analysis performed by Student's $t$-test of the data are reported in Table 2 .

The mean PVI values of limbs affected with extended $R$ of the GSV in the thigh and in the leg or detected only in the leg were significantly higher than the ones detected in normal limbs $(\mathrm{P}<0.01)$ and in the limbs with GSV incompetence detected only above the knee $(\mathrm{P}<0.05)$. The VPI values of the limbs with incompetent PVs were significantly higher in comparison with the ones with competent PVs $(\mathrm{P}<0.01)$.

The distribution of the VPI values obtained on the PTV district is shown in Figure 3. The results of the statistical analysis performed by Student's $t$-test of the data are reported in Table 3.

The mean PVI values appeared to be significantly higher in the majority of the cases with incompetent PVs, with or without GSV R $(\mathrm{P}<0.01)$. No relation between GSV R and venous hypertension of the PTV was found.

The mean values of PVI measured in the incompetent SSV
Table 5. Statistical Analysis of the Ambulatory PVI Values of the GSV Measured After Ten Exercises Standing Position Analyzed by Student's $t$-Test

\begin{tabular}{llllll}
\hline \multirow{2}{*}{ GSV } & \multicolumn{5}{c}{ Student's $t$-test values } \\
\cline { 2 - 6 } & IAK & IBK & TI & IPV & CPV \\
\hline C & 0.31 & 0.36 & 0.31 & 0.35 & 0.30 \\
IAK & & 0.26 & 0.94 & 0.42 & 0.66 \\
IBK & & & 0.23 & 0.81 & 0.11 \\
TI & & & & 0.42 & 0.56 \\
IPV & & & & & 0.23 \\
\hline
\end{tabular}

C: competent; IAK: incompetent above knee; IBK: incompetent below the knee; TI: totally incompetent; TI-IPV: totally incompetent and incompetent perforating veins; TI-CPV: totally incompetent and competent perforating veins.

were higher of the $58.3 \%$ in comparison with normal limbs (P $<0.01$ ), while the combination with incompetent PVs did not change the values (Fig. 4, Table 4).

\section{Ambulatory PVI}

The differential value observed between the standing VPI and the ambulatory PVI in the three examined districts was calculated. No significant difference in the GSV district, with

\section{Greater Saphenous Vein (GSV) Ambulatory VPI}

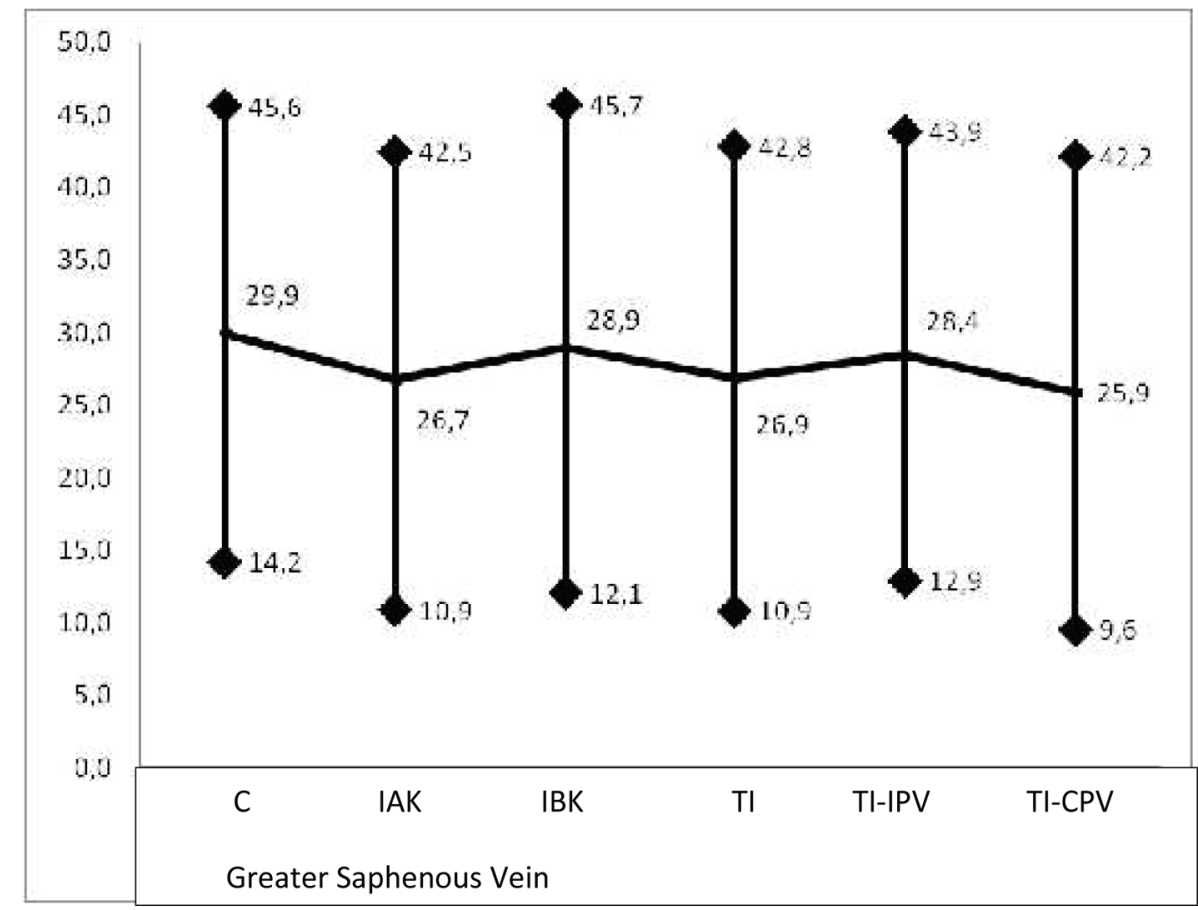

Figure 5. Ambulatory VPI values of the GSV measured after ten exercises in standing position. C: Competent; IAK: incompetent above knee; IBK: incompetent below knee; TI: totally incompetent; TI-IPV: totally incompetent and incompetent perforating veins; TI-CPV: totally incompetent and competent perforating veins. 
POSTERIOR TIBIAL VEIN AMBULATORY VPI

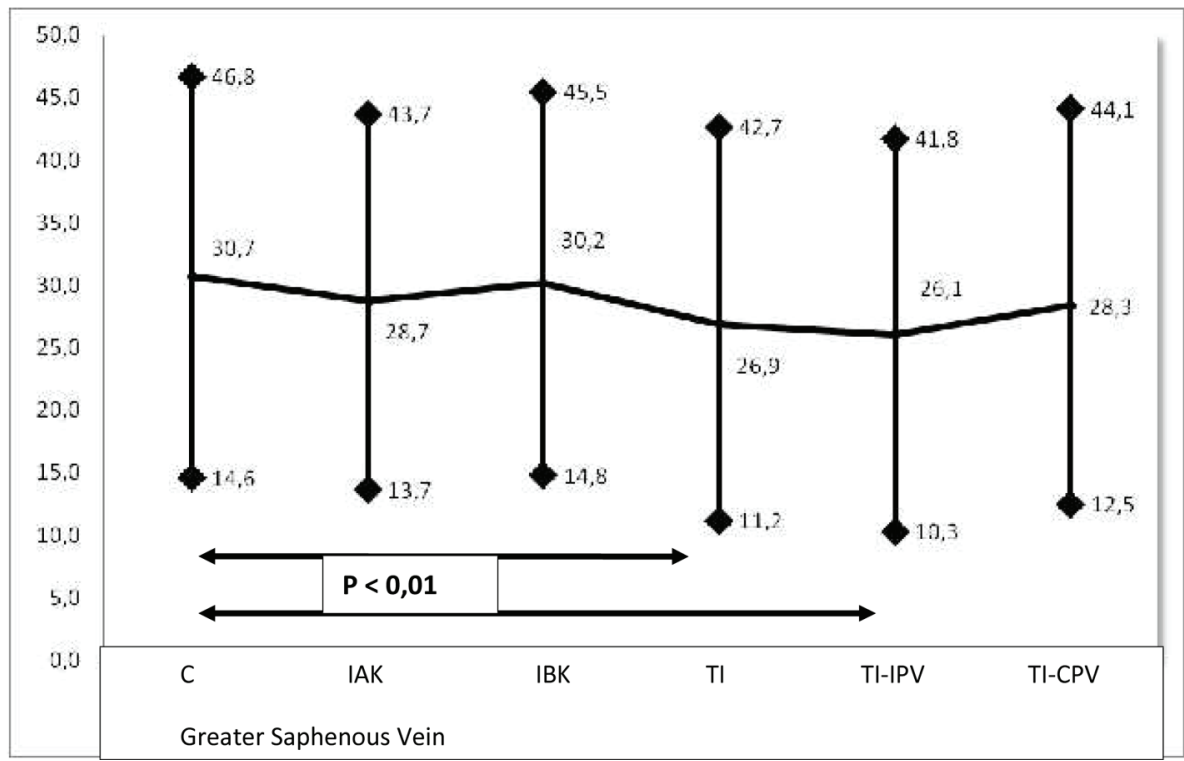

Figure 6. The presence of $R$ into the PV appears to be related with a decreased differential VPI measured in the PTV. The exclusion of patients free from IPV the differential VPI remains higher and the difference observed is not significantly different in comparison with the control group.

or without PV incompetence, in comparison with the normal ones, was found (Fig. 5, Table 5).

The mean PVI values measured in the PTV district appeared significantly lower $(\mathrm{P}<0.01)$ in comparison with the normal ones when the GSV R was combined with PV incompetence. An isolated R of the GSV did not influence the mean values of the ambulatory VPI into the deep system (Fig. 6, Table 6).

The differential VPI of the SSV was measured in a small number of cases, and therefore it was not analyzed.

\section{Statistical analysis}

The VPI values measured in the GSV, PTV and SSV in standing position and after 10 tip-toeing exercises were analyzed by Student's $t$-test and are reported in Figures and Tables 2-6.
The mean value of the standing VPI observed in the GSV, SSV and PTV of 162 normal subjects were analyzed by Student's $t$-test and corresponded to the mean value of $65 \mathrm{~mm} \mathrm{Hg}$ (range 37.6 - 93) (Fig. 2-4).

The distribution of the VPI detected in the GSV of the limbs with incompetent GSV and in competent GSV is shown in Figure 7. The details of the Bayesian test are reported in Table 7.

The statistic Bayesian test was performed in 1,044 limbs. Sensibility, specificity, the positive predictive value and the negative predictive value were calculated taking into consideration the VPI value of $80 \mathrm{~mm} \mathrm{Hg}$ as resulting by the Bayesian calculator (Microsoft Excel). The higher VPI peak of frequency corresponded to $60 \mathrm{~mm} \mathrm{Hg}$ in the limbs with competent GSV, while in the limbs with incompetent GSV a peak comprised between 90 and $100 \mathrm{~mm} \mathrm{Hg}$ was observed. The Bayesian analysis demonstrated that VPI measurement is a highly

Table 6. Statistical Analysis of the Ambulatory PVI Values of the PTV Measured After Ten Exercises Standing Position Analyzed by Student's $t$-Test

\begin{tabular}{llllll}
\hline \multirow{2}{*}{ GSV } & \multicolumn{5}{c}{ Student's $t$-test values } \\
\cline { 2 - 5 } & GSV RAK & GSV RBK & GSV RC & GSV RPV & GSV RC WRPV \\
\hline GSV C & 0.15 & 0.79 & 0.00097 & 0.00069 & 0.15 \\
GSV IAK & & 0.52 & 0.25 & 0.12 & 0.85 \\
GSV IBK & & 0.13 & 0.07 & 0.45 \\
GSV TI & & & 0.57 & 0.43 \\
GSV IPV & & & & 0.24 \\
\hline
\end{tabular}

C: competent; IAK: incompetent above knee; IBK: incompetent below the knee; TI: totally incompetent; TI-IPV: totally incompetent and incompetent perforating veins; TI-CPV: totally incompetent and competent perforating veins. 


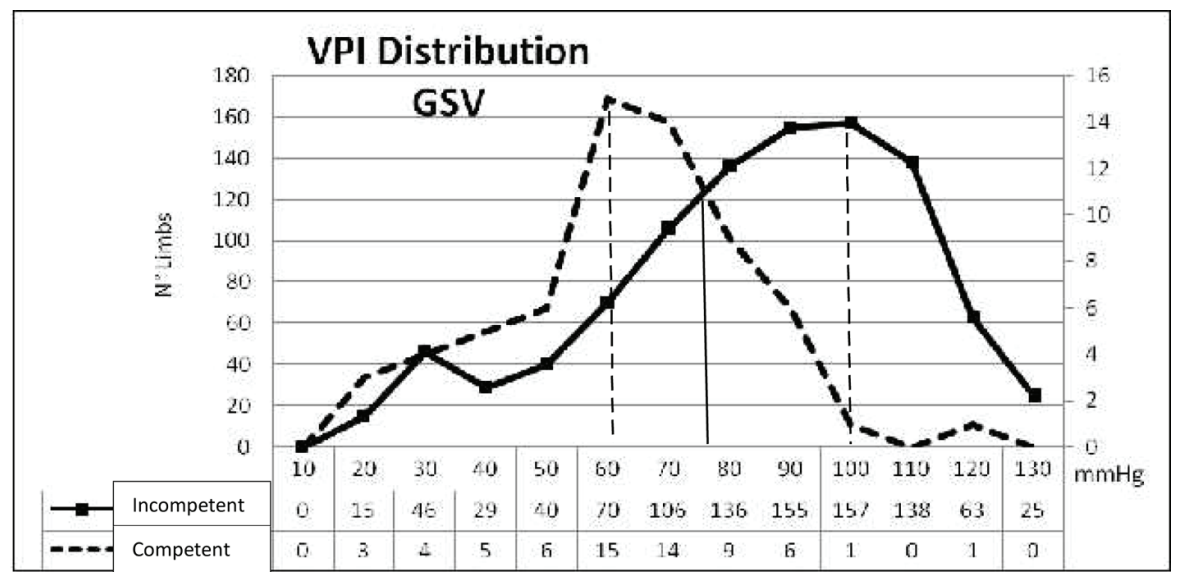

Figure 7. VPI distribution in the GSV of the limbs with incompetent and competent GSV. The statistic Bayesian test was performed in 1,044 limbs. Sensibility, specificity, the positive predictive value and the negative predictive value were calculated taking into consideration the VPI value of $80 \mathrm{~mm} \mathrm{Hg}$ as resulting by the Bayesian calculation. The higher VPI peak of frequency corresponds to $60 \mathrm{~mm} \mathrm{Hg}$ in the limbs with competent GSV, while in the limbs with incompetent GSV a peak comprised between 90 and $100 \mathrm{~mm} \mathrm{Hg}$ was calculated.

sensible examination: VPI $>80 \mathrm{~mm} \mathrm{Hg}$ is related with high probability of incompetent GSV. At the opposite, specificity is very low. The low positive predictive value demonstrates that a high VPI is not the expression of GSV incompetence. Therefore, other causes are to be invoked for explaining a GSV incompetence and high VPI. A high negative predictive value indicates that in limbs with VPI $>80 \mathrm{~mm} \mathrm{Hg}$ the probability to be related with GSV incompetence is very high.

The distribution of VPI in limbs with incompetent and competent SSV is shown in Figure 8. The statistic Bayesian test was performed in 1,044 limbs. Sensibility, specificity, the

Table 7. Statistical Analysis by Bayesian Test of VPI Measured in Competent and Incompetent GSV

\begin{tabular}{|c|c|c|c|c|}
\hline \multicolumn{5}{|c|}{ Bayesian test VPI } \\
\hline VPI & & C $0-1$ & & C 2-6 \\
\hline$\leq 80$ & a & 56 & $\mathrm{~b}$ & 442 \\
\hline$>80$ & c & 8 & d & 538 \\
\hline \multicolumn{2}{|c|}{ CGSV VPI $<80 \mathrm{~mm} \mathrm{Hg}$} & 56 & \multicolumn{2}{|l|}{$\mathrm{a}$} \\
\hline \multicolumn{2}{|c|}{ IGSV VPI > 80} & 442 & \multicolumn{2}{|l|}{$\mathrm{b}$} \\
\hline \multicolumn{2}{|c|}{ CGSV VPI $<80$} & 538 & \multicolumn{2}{|l|}{ d } \\
\hline \multicolumn{2}{|c|}{ CGSV VPI $>80$} & 8 & \multicolumn{2}{|l|}{$\mathrm{c}$} \\
\hline \multicolumn{2}{|l|}{ Total } & 1,044 & \multicolumn{2}{|c|}{$a+b+c+d$} \\
\hline \multicolumn{3}{|l|}{ Sensibility } & \multicolumn{2}{|c|}{$0.8750 \mathrm{a} /(\mathrm{a}+\mathrm{c})$} \\
\hline \multicolumn{3}{|l|}{ Specificity } & \multicolumn{2}{|c|}{$0.5490 \mathrm{~d} /(\mathrm{b}+\mathrm{d})$} \\
\hline \multicolumn{3}{|c|}{ Pos. predictive value } & \multicolumn{2}{|c|}{$0.1124 \mathrm{a} /(\mathrm{a}+\mathrm{b})$} \\
\hline \multicolumn{3}{|c|}{ Neg. predictive value } & \multicolumn{2}{|c|}{$0.9853 \mathrm{~d} /(\mathrm{c}+\mathrm{d})$} \\
\hline
\end{tabular}

The Statistic Bayesian test was performed in 1,044 limbs. Sensibility, specificity, the positive predictive value and the negative predictive value were calculated taking into consideration the VPI value of $80 \mathrm{~mm} \mathrm{Hg}$ as resulting by the Bayesian calculator (Fig. 7). C 0-1, C 2-6 of C.E.A.P. classification. positive predictive value and the negative predictive value were calculated taking into consideration the VPI value of 70 $\mathrm{mm} \mathrm{Hg}$ as resulting by the Bayesian calculator (Microsoft Excel). The details of the Bayesian test are reported in Table 8 .

In only three limbs R free (competent SSV) the VPI is > $70 \mathrm{~mm} \mathrm{Hg}$. In limbs with incompetent SSV, it is possible to find any VPI value homogeneously distributed. This test demonstrates that no R can be found in SSV of limbs with VPI $<$ $70 \mathrm{~mm} \mathrm{Hg}$. At the opposite, the presence of VPI $>70 \mathrm{~mm} \mathrm{Hg}$ is always related with SSV incompetence.

The distribution of VPI in the PTV of limbs with incom-

Table 8. Statistical Analysis by Bayesian Test of VPI Measured in Competent and Incompetent SSV

\begin{tabular}{|c|c|c|c|c|}
\hline \multicolumn{5}{|c|}{ Bayesian test SSV } \\
\hline VPI & & $\mathrm{C} 0-1$ & & C 2-6 \\
\hline$<70$ & a & 48 & $\mathrm{~b}$ & 309 \\
\hline$\geq 70$ & $\mathrm{c}$ & 3 & $\mathrm{~d}$ & 437 \\
\hline \multicolumn{2}{|c|}{ CSSV VPI $<70 \mathrm{~mm} \mathrm{Hg}$} & 48 & $\mathrm{a}$ & \\
\hline \multicolumn{2}{|c|}{ ISSV VPI $>70$} & 309 & $\mathrm{~b}$ & \\
\hline \multicolumn{2}{|c|}{ CSSV VPI $<70$} & 437 & $\mathrm{~d}$ & \\
\hline \multicolumn{2}{|c|}{ CSSV VPI $>70$} & 3 & $\mathrm{c}$ & \\
\hline \multicolumn{2}{|l|}{ Total } & 797 & $a+b+c$ & $+d$ \\
\hline \multicolumn{2}{|l|}{ Sensibility } & & 0.9412 a & $a /(a+c)$ \\
\hline \multicolumn{2}{|c|}{ Specificity } & & $0.5858 \mathrm{~d}$ & $\mathrm{~d} /(\mathrm{b}+\mathrm{d})$ \\
\hline \multicolumn{3}{|c|}{ Pos. predictive value } & 0.1345 a & $a /(a+b)$ \\
\hline \multicolumn{3}{|c|}{ Neg. predictive value } & $0.9932 \mathrm{~d}$ & $\mathrm{~d} /(\mathrm{c}+\mathrm{d})$ \\
\hline
\end{tabular}

The statistic Bayesian test was performed in 1,044 limbs. Sensibility, specificity, the positive predictive value and the negative predictive value were calculated taking into consideration the VPI value of $70 \mathrm{~mm}$ $\mathrm{Hg}$ as resulting by the Bayesian calculator (Fig. 8). C 0-1, C 2-6 of C.E.A.P. classification. 


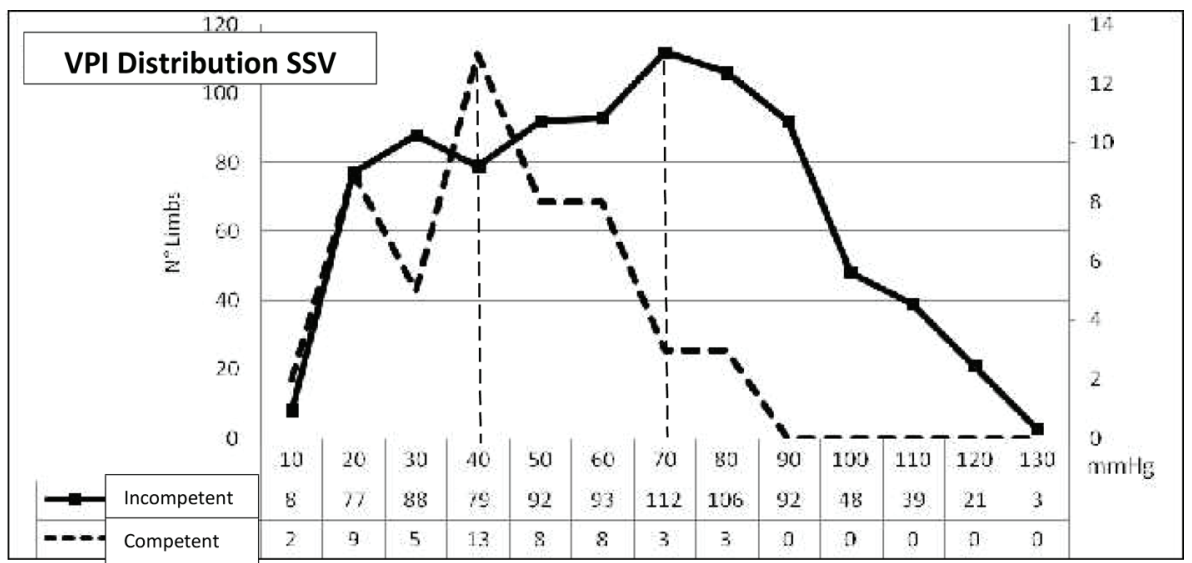

Figure 8. VPI distribution in the SSV of limbs with incompetent and competent SSV. The statistic Bayesian test was performed in 1,044 limbs. Sensibility, specificity, the positive predictive value and the negative predictive value were calculated taking into consideration the VPI value of $70 \mathrm{~mm} \mathrm{Hg}$ as resulting by the Bayesian calculation. In only three limbs R free and competent SSV the VPI > $70 \mathrm{~mm} \mathrm{Hg}$.

petent and competent GSV is shown in Figure 9. The statistic Bayesian test was performed in 1,044 limbs. Sensibility, specificity, the positive predictive value and the negative predictive value were calculated taking into consideration the VPI value of $80 \mathrm{~mm} \mathrm{Hg}$ as resulting by the Bayesian calculator (Microsoft- Excel). Two peaks of VPI are visible. The first one corresponding to a maximum of $60 \mathrm{~mm} \mathrm{Hg}$ in limbs with competent GSV and the second corresponding to a maximum of $80 \mathrm{~mm}$ $\mathrm{Hg}$ in limbs with incompetent GSV. In only one patient $\mathrm{R}$ free the PVI was more of $90 \mathrm{~mm} \mathrm{Hg}$. The details of the Bayesian test are reported in Table 9.

This test demonstrates that no $\mathrm{R}$ can be found in the GSV

Table 9. Statistical Analysis by Bayesian Test of VPI Measured in PTV of Limbs With Competent and Incompetent GSV

\begin{tabular}{|c|c|c|c|c|}
\hline \multicolumn{5}{|c|}{ Bayesian test PTV } \\
\hline VPI & & $\mathrm{C} 0-1$ & & C $2-6$ \\
\hline$<80$ & $\mathrm{a}$ & 53 & $\mathrm{~b}$ & 484 \\
\hline$\geq 80$ & $\mathrm{c}$ & 10 & d & 483 \\
\hline \multicolumn{2}{|c|}{ CSSV VPI $<80 \mathrm{~mm} \mathrm{Hg}$} & 53 & a & \\
\hline \multicolumn{2}{|c|}{ ISSV VPI $>80$} & 484 & $\mathrm{~b}$ & \\
\hline \multicolumn{2}{|c|}{ CSSV VPI $<80$} & 483 & $d$ & \\
\hline \multicolumn{2}{|c|}{ CSSV VPI $>80$} & 10 & $\mathrm{c}$ & \\
\hline \multicolumn{2}{|l|}{ Total } & 1,030 & $a+b+c$ & $c+d$ \\
\hline \multicolumn{2}{|l|}{ Sensibility } & & 0.8413 & $\mathrm{a} /(\mathrm{a}+\mathrm{c})$ \\
\hline \multicolumn{2}{|l|}{ Specificity } & & 0.4995 & $\mathrm{~d} /(\mathrm{b}+\mathrm{d})$ \\
\hline \multicolumn{3}{|c|}{ Pos. predictive value } & 0.0987 & $a /(a+b)$ \\
\hline \multicolumn{3}{|c|}{ Neg. predictive value } & 0.9797 & $\mathrm{~d} /(\mathrm{c}+\mathrm{d})$ \\
\hline
\end{tabular}

The statistic Bayesian test was performed in 1,044 limbs. Sensibility, specificity, the positive predictive value and the negative predictive value were calculated taking into consideration the VPI value of $80 \mathrm{~mm}$ $\mathrm{Hg}$ as resulting by the Bayesian calculator (Fig. 9). C 0-1, C 2-6 of C.E.A.P. classification. of limbs with VPI into the PTV $<80 \mathrm{~mm} \mathrm{Hg}$. At the opposite, the presence of VPI into the PTV $>80 \mathrm{~mm} \mathrm{Hg}$ is always related with GSV incompetence.

A significant correlation between VPI and the $\mathrm{C}$ of C.E.A.P. of every single limb was found and described in our previous paper $[10](\mathrm{P}<0.05-0.0001)$.

\section{Discussion}

Many efforts were done in the past and in the present for correctly defining the concept of CVD and chronic venous insufficiency (CVI) of the LL. Many descriptions of the pathophysiological mechanisms which can lead to CVI were also discussed, and the main factor which was often invoked was venous hypertension. However, no indications for a systematic VPM clinical application nor analyzed data can be found in the literature except for the noninvasive Doppler method. The studies performed by Bartolo et al [3,4] clearly indicate how significant and useful can be the VPI measurement in venous pathophysiology and in the clinical practice.

Unfortunately, in spite of the above mentioned research and concepts, it is possible to read in the literature various sentences and definitions as "venous hypertension, venous insufficiency, iperpression (French), venous congestion, hemodynamic worsening or improvement, VVLL at "high or low pressure" and many others. None of them is sufficiently supported by a convincing hemodynamic instrumental evaluation except for those which were subjected to invasive and noninvasive VPM and/or plethysmographic examinations performed in specialized centers $[3-5,7,8,15,16]$.

On the other hand, it must be noted that very small importance was given to the invasive VPM in the past and in more recent guidelines [6]. In recent reports, some perplexities concerning the invasive method can be found and the VPM did not appear to be essential and/or reliable in the diagnostic procedures commonly performed for CVD [8, 17]. The invasive method for the ambulatory VPM, which has been consid- 


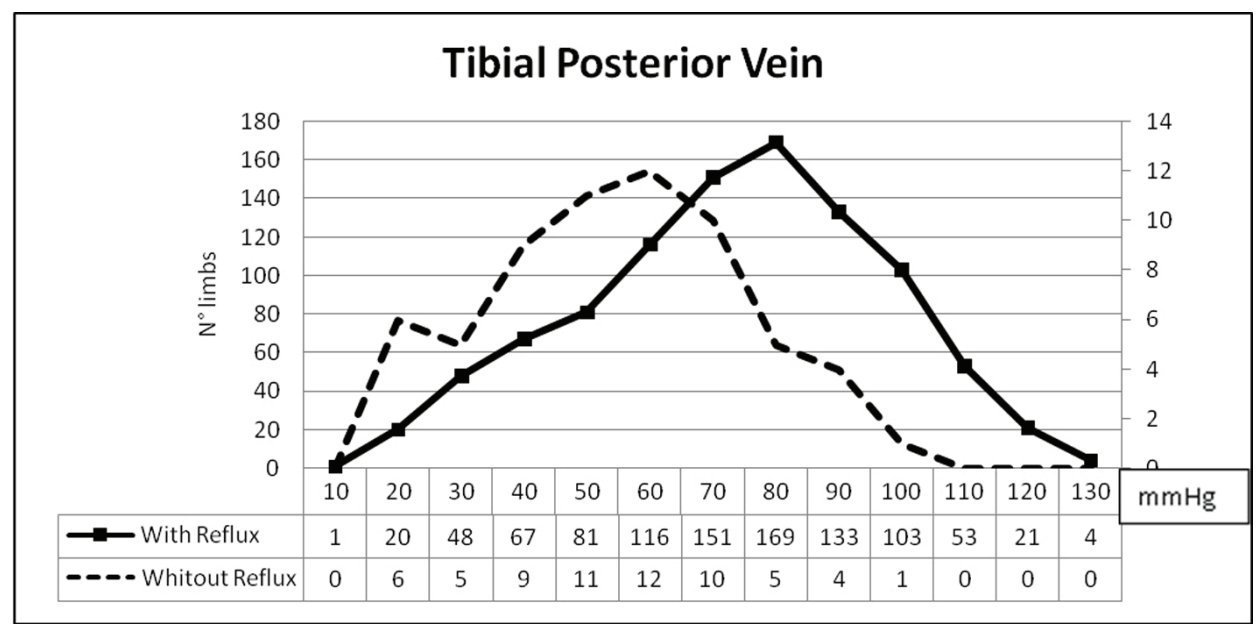

Figure 9. VPI distribution in the PTV of limbs with incompetent and competent GSV. The statistic Bayesian test was performed in 1,044 limbs. Sensibility, specificity, the positive predictive value and the negative predictive value were calculated taking into consideration the VPI value of $80 \mathrm{~mm} \mathrm{Hg}$ as resulting by the Bayesian calculation. Two peaks of VPI are visible. The first one corresponding to a maximum of $60 \mathrm{~mm} \mathrm{Hg}$ in limbs with competent GSV and the second corresponding to a maximum of $80 \mathrm{~mm}$ $\mathrm{Hg}$ in limbs with incompetent GSV.

ered until now the gold standard, has been more recently found not always accurate in detecting changes in the pressure of the tibial and popliteal veins as in the past [17]. It seems also quite obvious that a systematic invasive VPM cannot be nowadays proposed to patients. It has also been confirmed that DUS investigation is the most useful test for detecting and localizing chronic venous obstruction and valvular incompetence. However, it provides relatively little quantitative hemodynamic information and is often combined with measurements of hemodynamic severity determined, at the present time, only by a number of plethysmographic methods [8].

Coming back to our experience, we can affirm that it is possible to perform systematically every kind of noninvasive vascular diagnostics, often composed by a detailed DUS examination, VPI measurement and/or photo-plethysmography. This made it possible to acquire a large experience which showed how useful is a more accurate morphologic and hemodynamic information concerning the pathophysiological condition of every single case. This experience seems to be helpful for correctly defining the conceptual relation between CVD and CVI. The latter should be represented by the combination of clinical severity, time of pathological $\mathrm{R}$ and venous hypertension which can be easily evaluated by the VPI measurement. The data emerging from plethysmography $[8,15,16]$ can be extremely useful for detecting the combination of CVD with the venous pump insufficiency due to muscular, joints and skeletal alterations. It seems quite difficult to define all the clinical and hemodynamic conditions as CVI, when all the above mentioned parameters are not combined.

The several diameters were calculated for clinical purposes, but the resulting values were not correlated with the VPI measured in the various districts nor analyzed. The significance of venous diameters in superficial CVD of the LL was previously described in several papers by N. Labropoulos. Here we mention a recent publication [18].

The present study demonstrated that it is possible to de- termine the functional condition of different venous districts as the deep system (PTV) and the superficial one (GSV-SSV) by the simple measurement of the VPI in standing position. The dynamic measurement after exercise did not produce significant results, and it appeared sometimes very difficult to be performed in the elder patients, especially for those who were affected with bone and joints pathology, in the cases with chronic painful ulcers and in not compliant patients.

The systematical investigation by Doppler standing VPI measurements can also show and explain how the two systems can influence one each other and, sometimes, which role is playing the proximal and/or distal R of the GSV and of the incompetent PVs, so that it is possible to understand whether they are to be surgically eliminated, interrupted or preserved. As it can be easily understood from Figure 3, a GSV hypertension can increase the pressure in competent PTV. This phenomenon is more evident when PVs of the leg are also incompetent. It is now possible to better define the so called "overload syndrome" which was suspected in the past during previous studies [19] and was often mentioned during various meetings, but never published.

The ambulatory VPI measurements seemed to be helpful in the few cases affected with deep CVI, in the occlusive venous disease, which were not taken into consideration owing to the low number of cases. Nevertheless the statistical analysis of ambulatory VPI values measured in our cases affected with superficial CVD appeared to be of a low significance as previously communicated by other authors who performed invasive [17] and noninvasive [3, 4] VPM. In some of our cases with superficial R combined with incompetent PVs the standing pressure measured in the PTV was significantly higher (Fig. 3, Table 3) in confirmation of the "ankle blow out syndrome" described by Cockett and Jones [20]. At the opposite, in the same cases (GSV R + incompetent PVs) the ambulatory VPI of PTV was significantly lower (Fig. 6, Table 6). This observation seems to confirm that the venous muscular pump 
efficiency can lead to normalization of the deep venous hypertension caused by an "overload syndrome", i.e., secondary to superficial R.

During the previous experience, which is still in progress in our clinical practice, many advantages in the prophylactic and therapeutic project of every single patient were found. First of all, it seems to be quite reliable that patients with a well-defined CVI have thrombotic risk factors being increased in relation with the severity of the disease detected by the VPI values and not only by means of clinical judgment [10]. It seems also logical to divide the patients, who are candidate to surgical procedures for varicose veins of the LL, into different groups of priority on the basis of such risk factors. A high value of VPI in limbs affected with incompetent GSV must lead the investigators to search for a long $\mathrm{R}$ in the GSV and for the presence of incompetent PVs in order to extend the surgical or endovascular treatment to the venous districts responsible for venous hypertension with the main purposes of bringing them back to normal hemodynamic conditions, reducing risks and symptoms and the postoperative recurrent rate. The previous studies [3, 4] have already demonstrated that the VPI of the limbs subjected to the surgical ablation of the incompetent GSV and/or SSV were significantly reduced, therefore, it can be assumed that such hemodynamic approach may be applied even for the patient's follow -up, exactly as plethysmography.

In cases affected with early superficial venous disease, with initial $\mathrm{R}$ and limited varicose veins, the VPI measurement can represent a useful parameter for the choice of minimally invasive procedures and for monitoring the disease progression while patients are subjected to conservative treatments. Our experience also demonstrated that VPI does not always correspond to the clinical class of the disease. Therefore, it can be affirmed that such a parameter can easily make it possible to calculate the mean counter pressure necessary in every single limb and, consequently, to chose the more proper class of compression of elastic stockings more than by a simple clinical criterion [10].

\section{Conclusions}

It appears to be advisable to perform systematically the VPI measurements by noninvasive method in all the cases affected with CVD. When the diagnostic findings clearly indicate the combination between morphological alterations, $\mathrm{R}$ and hypertension, it seems more proper to define the disease as CVI. Noninvasive VPI measurements represent one of the more simple and repeatable methods to achieve the hemodynamic information which makes possible a more precise selection and follow-up of the patients affected with CVD/CVI. Standing VPI appeared to be a significant evaluation, and it is the expression of the consequences of the functional disorder detected in the various venous districts. $\mathrm{R}$ in GSV at the leg and in incompetent PV increases VPI and is strictly related with the severity of the disease. Superficial venous $\mathrm{R}$ and hypertension can also lead to secondary deep venous hypertension, and therefore, increase the severity of the disease. The mean VPI standing values detected in every single limb, compared with the mean normal values, is a highly predictive diagnostic and prognos- tic investigation and gives a great help for any therapeutic decision in the clinical practice as surgery, endovascular treatments, sclerotherapy, physical therapy and patient's follow-up.

\section{Acknowledgement}

This study was supported by CZ Medicali, Cuggiorno (MI), Italy.

\section{References}

1. Hojensgard IC, Sturup H. Static and Dynamic Pressures in Superficial and Deep Vein of the Lower Extremity in Man. Acta Phys. Scandinav 1952.

2. Gaylis H. Some observations on peripheral venous pressure using a non-invasive technique: a preliminary report. Br J Surg. 1975;62(4):259-263.

3. Bartolo M. Phlebodopplertensiometry, a non invasive method for measuring venous pressure. Folia Angiol. 1977;25:199-203.

4. Bartolo M, Nicosia PR, Antignani PL, et al. Noninvasive venous pressure measurements in different venous diseases. Angiology. 1983;34.

5. Nicolaides AN. Investigation of Chronic Venous Insufficiency: A Consensus Statement. Circulation. 2000;102: e126-e163.

6. Collegio Italiano di Flebologia. Linee guida diagnostico terapeutiche delle malattie delle vene e dei linfatici. Acta Phlebologica. 2003;4(1-2).

7. Lattimer CR, Azzam M, Kalodiki E, Makris GC, Geroulakos G. Compression stockings significantly improve hemodynamic performance in post-thrombotic syndrome irrespective of class or length. J Vasc Surg. 2013;58(1):158-165.

8. Meissner MH, Moneta G, Burnand K, Gloviczki P, Lohr JM, Lurie F, Mattos MA, et al. The hemodynamics and diagnosis of venous disease. J Vasc Surg. 2007;46(Suppl S):4S-24S.

9. Spina T, Corcos L, Peruzzi G, Baldelli G, De Anna D. The value of Doppler venous pressure index in the diagnosis of chronic venous insufficiency of the lower limbs. International. Angiology. 2009;28(Suppl.1 4):92.

10. Corcos L, Pontello D, Spina T. Elastic compression treatment of chronic superficial venous insufficiency of the lower limbs based on Doppler venous pressure index measurements. Veins \& Lymphatics. 2015;4(2230):2-6.

11. Cataldo JL, de Godoy JM, de Barros N. The use of compression stockings for venous disorders in Brazil. Phlebology. 2012;27(1):33-37.

12. van Bemmelen PS, Bedford G, Beach K, Strandness DE. Quantitative segmental evaluation of venous valvular reflux with duplex ultrasound scanning. J Vasc Surg. 1989;10(4):425-431.

13. Vasdekis SN, Clarke GH, Nicolaides AN. Quantification of venous reflux by means of duplex scanning. J Vasc Surg. 1989;10(6):670-677.

14. Bjordal J. Circulation patterns in incompetent perforating 
veins of the calf in venous dysfunction. In May R, Partsch H, Staubesand J. Perforating Veins. Urban \& Schwarzenberg. Muchen-Wien-Baltimore. 1981;71-88.

15. Fronek A. Noninvasive diagnostics in vascular disease. McGraw -Hill Book Company. U.S.A. 1989.

16. Schultz-Ehremburg U, Blazek V. Advances in computer-aided noninvasive vascular diagnostics. Dusseldorf : VDI-Verlag GmbH. 1994.

17. Neglen P, Raju S. Ambulatory venous pressure revisited. J Vasc Surg. 2000;31(6):1206-1213.
18. Labropoulos N, Kokkosis AA, Spentzouris G, Gasparis AP, Tassiopoulos AK. The distribution and significance of varicosities in the saphenous trunks. J Vasc Surg. 2010;51(1):96-103.

19. Corcos L, Peruzzi GP, Romeo V, Procacci T, Zamboni P, Dini S. [External valvuloplasty of the sapheno-femoral junction]. Phlebologie. 1991;44(2):497-508.

20. Cockett FB, Jones DE. The ankle blow-out syndrome; a new approach to the varicose ulcer problem. Lancet. 1953;1(6749):17-23. 